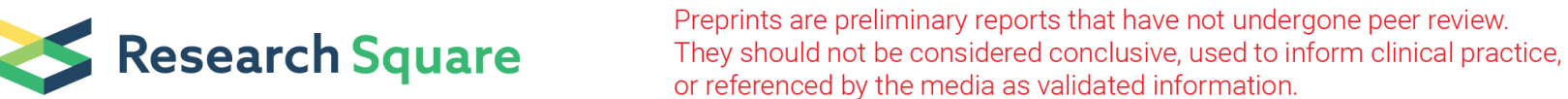 \\ Realization of Twenty Monochromatic Beams using Photonic Structure via Principle of Filtering
}

\section{K.P. Swain}

Gandhi Institute For Technological Advancement

\section{Subhankar Das}

Dai Hoc Duy Tan

\section{Soumya Ranjan Samal}

Technical University-Sofia: Tehniceski universitet-Sofia

\section{Sanjay Kumar Sahu}

Lovely Professional University

Gopinath Palai ( $\nabla$ gpalai28@gmail.com )

GITA, BHUBANESWAR https://orcid.org/0000-0002-9891-493X

\section{Research Article}

Keywords: Monochromatic signal, absorption-reflection-transmission, plane wave expansion, reflectance curve

Posted Date: July 26th, 2021

DOl: https://doi.org/10.21203/rs.3.rs-697176/v1

License: (c) (i) This work is licensed under a Creative Commons Attribution 4.0 International License.

Read Full License 


\title{
Realization of twenty Monochromatic beams using photonic structure via principle of filtering
}

\author{
K.P.Swain ${ }^{1}$, Subhankar Das ${ }^{2}$, Soumya Ranjan Samal ${ }^{3}$, Sanjay Kumar Sahu ${ }^{4}$, G.Palai ${ }^{1 *}$ \\ ${ }^{1 *}$ Department of Electronics and communication engineering, Gandhi Institute for \\ Technological Advancement (GITA)), Bhubaneswar, India, email: kaleep.swain@gmail.com, \\ 2. Researcher, Department: Honors Programme; Duy Tan University, Vietnam \\ (info.subhankardas@gmail.com) \\ 3. Faculty of Telecommunications, Technical University of Sofia, \\ Sofia, Bulgaria (sranjansamal@tu-sofia.bg) \\ 4. Lovely Professional University (sanjay.23393@1pu.co.in) \\ *Corresponding author; email-gpalai28@gmail.com, phone no. (+91)9439045946
}

\begin{abstract}
The current work employs silicon-based one dimensional photonic structure which delivers '20' different types of monochromatic beams (wavelengths) via filtering action. The I/P signals are essentially varies from visible to short infrared range to justify the work. Though similar type of works related to filtering application are found in the literature, the present research deals with an output signal which could be deployed in different purposes vis-à-vis dentistry, dermatology, spectroscopy, printing, holography, barcode scanning etc. The physicality of this work incorporates 68 layers of silicon monoxide and silicon based one-dimensional optical waveguide along with their configuration where the plane wave expansion method does fulfill the nitty-gritty of required mathematics to solve out electromagnetic wave equations. Reflectance and transmittance characteristics along with the absorbance are the critical parameters that substantiate the said application.
\end{abstract}

Keywords: Monochromatic signal, absorption-reflection-transmission, plane wave expansion, reflectance curve

\section{INTRODUCTION}

In the today's era research on photonics seems to be an exciting area because of its applicability in engineering and medical science. It is replacing many conventional or existing techniques to postulate new functional devices. For example; it can be swelled to networking, sensing, communication and many others. In fact, the twenty-five years of research on photonics bestows various types of new applications which are made available in the literature. A few of the same are highlighted here through some remarkable findings. For example; plasmonic $(\mathrm{ZnO})$ nano wire is investigated in the reference [1] to study the behavior of the periodic structure and it is observed that the diameter of lasing is reduced significantly by placing the plasmonic monochromatic in the periodic structure. Similarly, photonic crystal fiber based on $\mathrm{Si}_{7} \mathrm{~N}_{3}$ glass material is designed and analyzed in the reference [2] using the finite element method which is highly beneficial for optical coherence tomography and optical signal processing. Further in the reference [3], one-dimensional magnetized plasma photonic crystal is designed using a simple transfer matrix method for ultra-short pulse monochromatic application. Beside this, a review is also conducted on III-V monochromatic which have been integrated on the Si platform in the reference [4] to study the performance in the telecom field. Again, two-dimensional crystal is used to analyze a laser based acetylene sensor in reference [5] by FDTD method using MEEP software whereas a single photonic crystal structure is used to generate 16 laser beams in [6] which are predominantly used in medical field. Beside these, recently a series of work related to the silicon waveguide have been disclosed for various type of the application vis-a-vis MUX/DEMIX, monochromatic filter, locking and unlocking the device, VLC and robotic application [7-13].Even though one-dimensional photonic structure bestows a 
different notable applications [7-13] and capable of generating 16 types of monochromatic beams [6], we in this work generate twenty numbers of monochromatic beams in visible and near-infrared (NIR) regime using the principle of filteing . For examples; different signals of He-Cd, Argon, Krypton, He-Ne, Ruby, Nd:YAG, Tm:YAG, Ho:YAG, Er:YAG, Er:YSGG, and Iodine monochromatic beams are generated and their wavelengths are drawn from the reference[14]. The aforementioned monochromatic beams have extensively been used in the biomedical application especially in the field of (dentistry and soft tissue treatment) and scientific research as pronounced inFigure1.

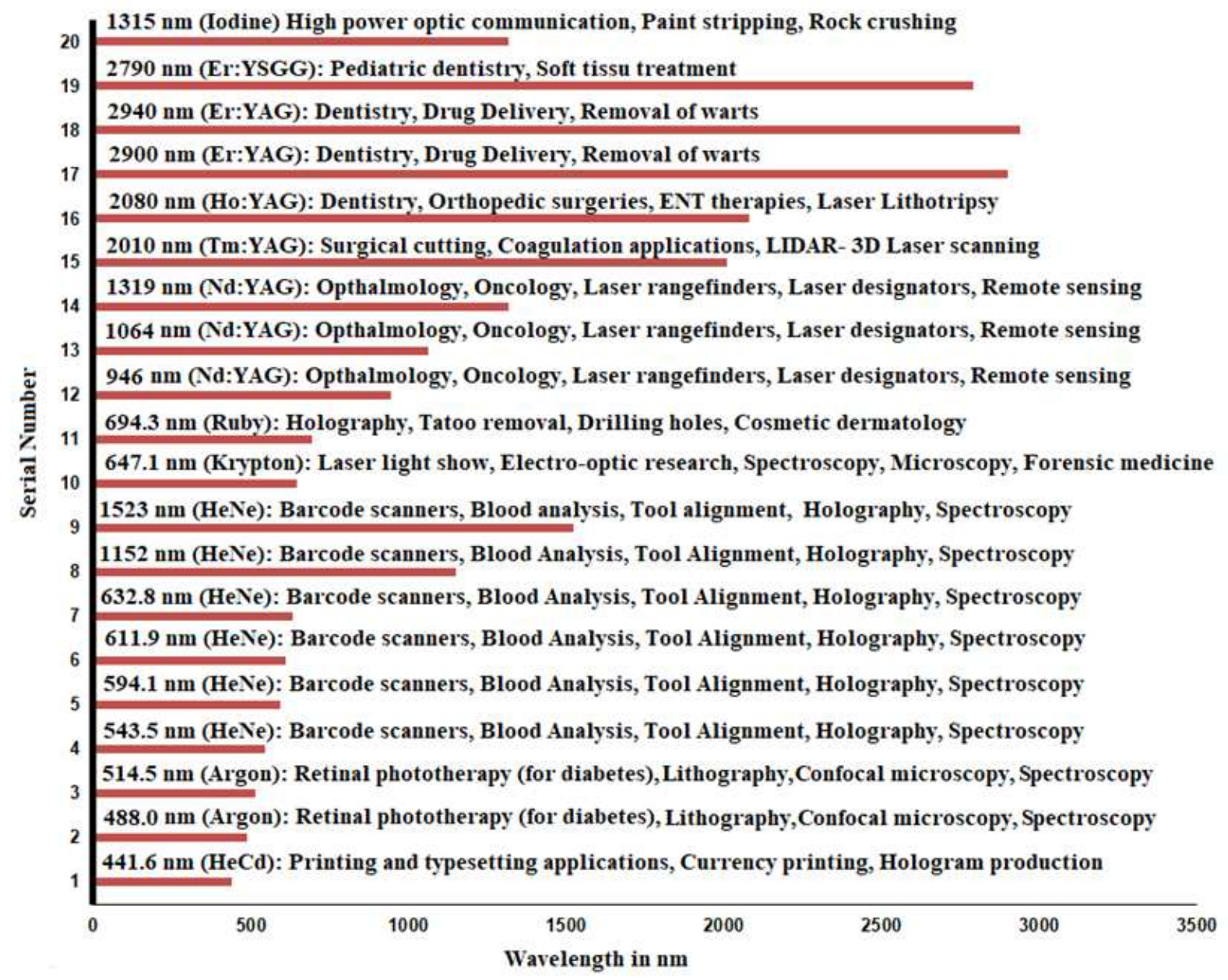

Fig. 1 Representation of Monochromatic beams along with their application [15]

In Figure 1, the vertical axis represents the serial number of monochromatic beams along with their diverse applications wherein wavelengths are represented in the horizontal axis. In this work, selection 2 discloses the structure and the operational mechanism whereas section 3 confined the relevant mathematics for the proposed structure. Further, the result and discussion are described in section 4 followed by the conclusions are made in section 5 .

\section{Structure and the operational mechanism}

The structure which is opted in the present research to divulge different monochromatic beams with respect to the visible to short infrared signal (input signal) is a simple and one-dimensional photonic structure which is made up of silicon monoxide and silicon material at the odd and even layer respectively. Moreover the numbers of layers are considered to be 68 , however, the defects are created at the $18^{\text {th }}$ and $52^{\text {nd }}$ position. Those two deffects are dealt with air instead of silicon material, which is indicated in Figure 2. 


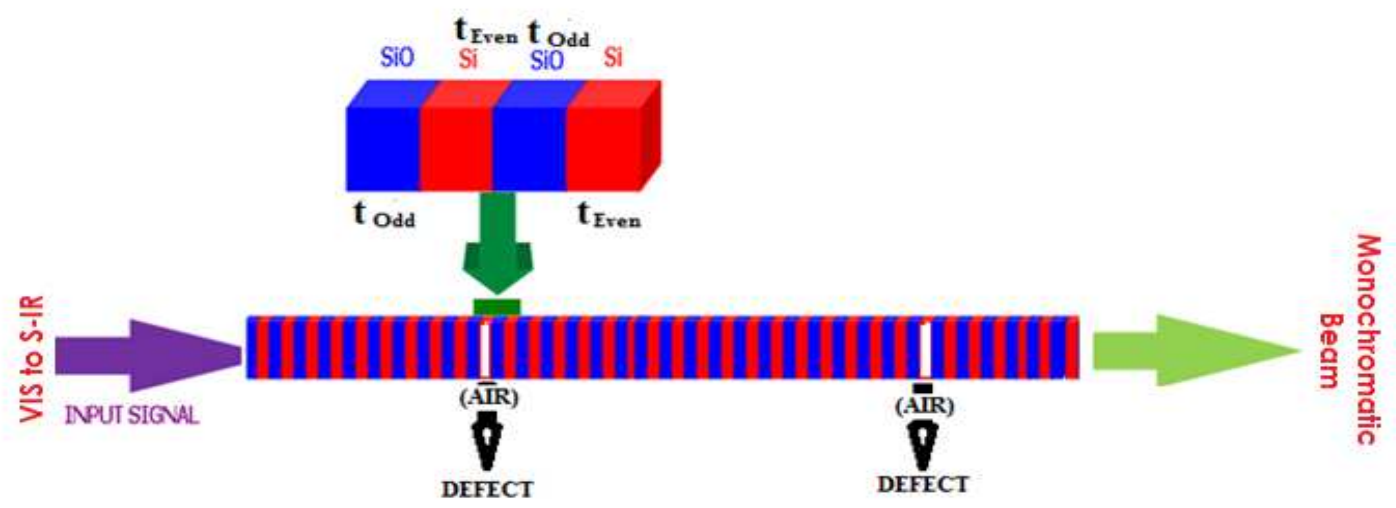

Fig. 2 Schematic diagram of 1D photonic structure for emerging monochromatic beams

Though figure 2 consists of 68 layers of silicon monoxide and silicon material, an inset corresponding to the four layers of the same is mentioned for the sake of better understanding, where $\mathrm{SiO}$ and $\mathrm{Si}$ are represented as silicon monoxide and silicon respectively. Similarly,todD and teVEN are represented as the thickness of odd and even layer respectively. Further moving to the realization of operational mechanism in the current research work, it realized that physics of the proposed works deal with refractive indices and thickness of the odd and even layers pertaining to the input signal where the mathematics of the works manipulate with the Helmholtz's differential equation along with the plane wave expansion method as well as numerical investigation.

\section{The mathematical formulation of the proposed structure}

The reflectance of the 1-D layer can be analyzed through the wave equation which is drawn from Maxwell's equation. The wave equation here governed by a second-order differential equation (Helmholtz equation) [16]and it can be written as;

$$
\frac{\partial^{2} E_{Z}(x)}{\partial x^{2}}+n^{2}(x) \frac{\omega^{2}}{c^{2}} E_{z}(x)=0
$$

Where E, n, $\omega$, and c be the electric field, refractive index, frequency, and velocity of light respectively

$$
E_{j}(x)=A_{j} e^{i . n_{j} k \cdot x_{j}}+B_{j} e^{-i . n_{j} k \cdot x_{j}}
$$

Where $\mathrm{A}$ and $\mathrm{B}$ are represented as the amplitudes of forward and backward waves and $x_{\mathrm{j}}$ is the coordinate of $\mathrm{j}^{\text {th }}$ interfaces, $\mathrm{k}$ is the propagation constant, which is ' $\omega / \mathrm{c}$ '. Before going to continue the value of A and $\mathrm{B}$, let us focus on the understanding of A (forward) and B (backward) of the signal in the proposed structure.

To find out the A and B, let us apply the suitable conditions at the interfaces between the consecutive layers and it can be written as (electric filed is a function of ' $x$ ')

$$
\begin{gathered}
E_{j}=E_{j+1} \\
\frac{\partial E_{j}}{\partial x}=\frac{\partial E_{j+1}}{\partial x}
\end{gathered}
$$


Combing equation (2) and (3), we get that

$$
\begin{aligned}
& A_{j} e^{i \cdot n_{j} k \cdot x_{j}}+B_{j} e^{-i \cdot n_{j} k \cdot x_{j}}=A_{j+1} e^{i \cdot n_{j+1} k \cdot x_{j}}+B_{j+1} e^{-i \cdot n_{j+1} k \cdot x_{j}} \\
& i . n_{j} \cdot k \cdot A_{j} e^{i \cdot n_{j} k \cdot x_{j}}-i \cdot n_{j} \cdot k \cdot B_{j} e^{-i \cdot n_{j} k \cdot x_{j}}=i \cdot n_{j+1} \cdot k \cdot A_{j+1} e^{i \cdot n_{j+1} k \cdot x_{j}}-i \cdot n_{j+1} \cdot k \cdot B_{j+1} e^{-i \cdot n_{j} k \cdot x_{j}}
\end{aligned}
$$

The above-said equation can be written in matrix form as

$$
\begin{aligned}
& \left(\begin{array}{cc}
A_{0} & B_{0} \\
i k n_{0} A_{0} & -i k n_{0} B_{0}
\end{array}\right)\left(\begin{array}{c}
e^{i k n_{0} x_{0}} \\
e^{-i k n_{0} x_{0}}
\end{array}\right)=\left(\begin{array}{cc}
A_{1} & B_{1} \\
i k n_{1} A_{1} & -i k n_{1} B_{1}
\end{array}\right)\left(\begin{array}{c}
e^{i k n_{1} x_{0}} \\
e^{-i k n_{1} x_{0}}
\end{array}\right) \\
& \left(\begin{array}{cc}
A_{1} & B_{1} \\
i k n_{1} A_{1} & -i k n_{1} B_{1}
\end{array}\right)\left(\begin{array}{c}
e^{i k n_{1} x_{1}} \\
e^{-i k n_{1} x_{1}}
\end{array}\right)=\left(\begin{array}{cc}
A_{2} & B_{2} \\
i k n_{2} A_{2} & -i k n_{2} B_{2}
\end{array}\right)\left(\begin{array}{c}
e^{i k n_{2} x_{1}} \\
e^{-i k n_{2} x_{1}}
\end{array}\right) \\
& \left(\begin{array}{cc}
A_{68} & B_{68} \\
i k n_{68} A_{68} & -i k n_{68} B_{68}
\end{array}\right)\left(\begin{array}{c}
e^{i k n_{68} x_{68}} \\
e^{-i k n_{68} x_{68}}
\end{array}\right)=\left(\begin{array}{cc}
A_{69} & B_{69} \\
i k n_{69} A_{69} & -i k n_{69} B_{69}
\end{array}\right)\left(\begin{array}{c}
e^{i k n_{69} x_{68}} \\
1
\end{array}\right)
\end{aligned}
$$

We solve sets of linear system equation, using Crammer's method. Further, the maximum wavelength corresponding to the refractive indices as well as thickness can be found by using the following expression.

$$
\lambda=2\left(n_{S i O} \cdot t_{S i O}+n_{S i} \cdot t_{S i}\right)
$$

Combining the Equation (5) and (6), the reflectance corresponding to the wavelength can be computed.

After the computation of reflectance, we move to compute the absorbance of the structure using the following Equations [17];

$$
\begin{gathered}
A_{\text {siliconmonoxide }}=e^{-33 \alpha_{\text {siliconmonoxide }} t_{\text {siliconmonoxide }} 7(\mathrm{a})} \\
A_{\text {silicon }}=e^{-33 \alpha_{\text {silicon }} t_{\text {silicon }}} \\
A_{\text {Air }}=e^{-2 \alpha_{\text {Air }}} t_{\text {air }} \\
\alpha=\frac{4 \pi n_{\text {imaginary }}}{\lambda_{\text {incident }}}
\end{gathered}
$$

Where equations 7(a), 7(b) and 7(c) are represented as the absorbance of silicon monoxide, silicon, and air (18 ${ }^{\text {th }}$ and $52^{\text {nd }}$ position) layer respectively. Further $\alpha$ is known as the absorption coefficient of the materials, which depends on the $n_{\text {imaginary }} a n d \lambda_{\text {incident }}$, which are the imaginary part of the refractive index and incident wavelength respectively.

Finally, the resultant absorbance of the 1D structure can be determined.

$$
\mathrm{A}=\mathrm{A}_{\text {silcion monoxide }} \times \mathrm{A}_{\text {silicon }} \times \mathrm{A}_{\text {Air }}
$$




\section{RESULT AND DISCUSSION}

The result and discussion of the present works depend on the analysis of the reflectance as well as absorbance of the proposed structure to understand the filtering application where the plane wave expansion method is employed to study the reflectance characteristics of 1-D silicon waveguide and absorbance with the help of numerical treatment. Further operational mechanism deals with the generation of the monochromatic beams pertaining to the visible and near-infrared regime and the generated wavelengths are $441.6 \mathrm{~nm}, 488.0 \mathrm{~nm}, 510.5 \mathrm{~nm}, 514.5 \mathrm{~nm}, 532.0 \mathrm{~nm}, 539.5$ $\mathrm{nm}, 543.5 \mathrm{~nm}, 578.2 \mathrm{~nm}, 594.1 \mathrm{~nm}, 611.9 \mathrm{~nm}, 632.8 \mathrm{~nm}, 647.1 \mathrm{~nm}, 694.3 \mathrm{~nm}$ of visible regime and the wavelength of Monochromatic beams are 946 nm, 1064 nm, 1152 nm, 1315 nm, 1319 nm, 1523 nm, 2010 nm, 2080 nm, 2790 $\mathrm{nm}, 2900 \mathrm{~nm}, 2940 \mathrm{~nm}$ of infrared regime respectively. The reasons for generating such wide range of wavelengths are that these wavelengths have been used for various purposes now-a-days

To obtain the reflectance characteristics of photonic structure pertaining to the proposed input signals, plane wave expansion (PWE) technique is used for the same. Even though the simulation has been done to generate twenty different monochromatic beams, here Figure 3(a) and 3(b) indicate the reflectance curve for Argon (Ar+) and Er:EAG signals only.
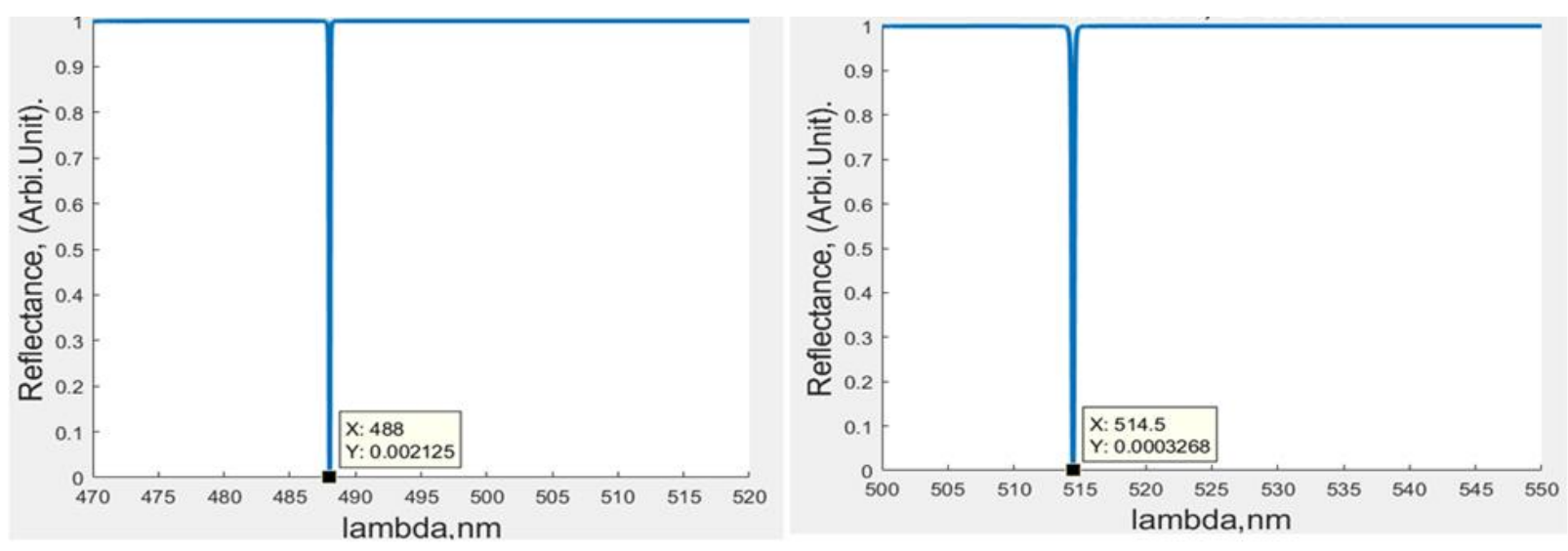

Fig. 3(a) Reflectance characteristics of 1D photonic structure for realizing argon monochromatic
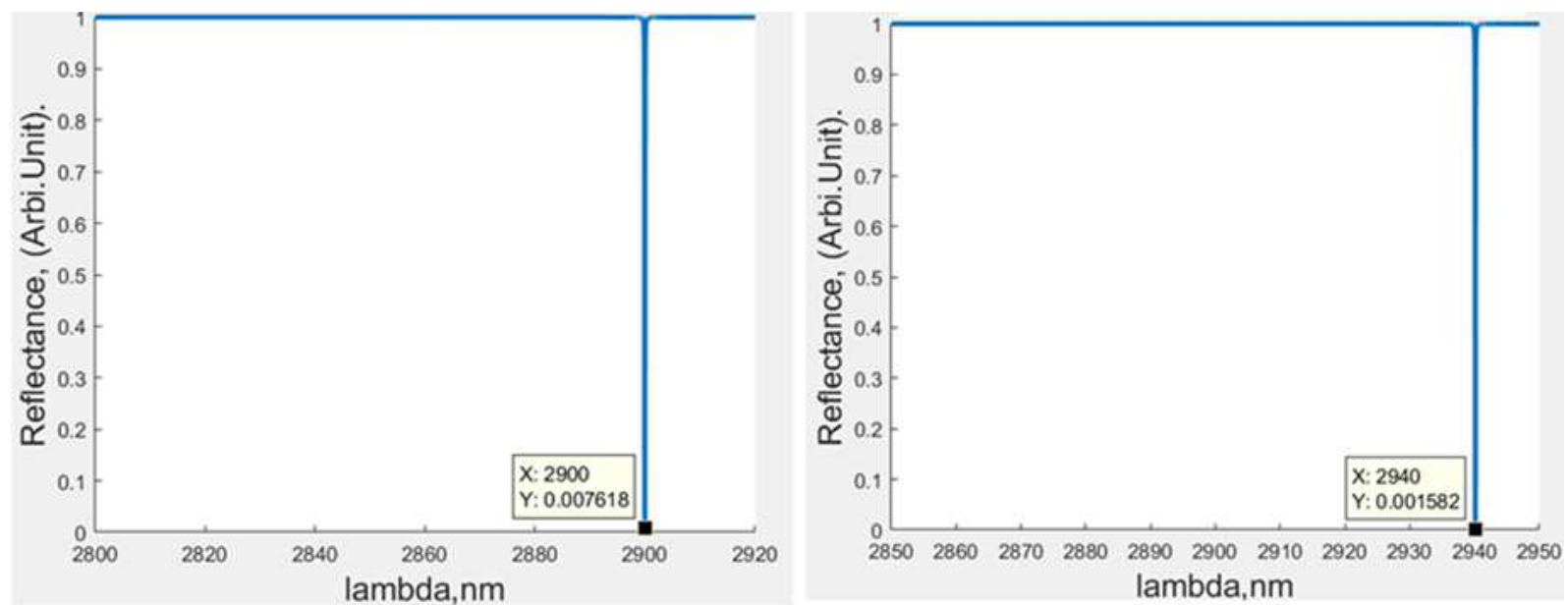

Fig. 3(b) Reflectance characteristics of 1D photonic structure for realizing Er:YAG monochromatic 
In Figure 3(a) and 3(b), the reflectance (arbi.unit) and wavelength (lambda, nm) are taken along vertical and horizontal axis respectively. Further analyzing these figures, it is found an interesting result with respect to the reflectance characteristics. From figure 3(a), it is revealed that almost $100 \%$ reflectance is obtained for the entire wavelengths excepting $488.0 \mathrm{~nm}$ and $514.5 \mathrm{~nm}$. Zero reflectance is exhibited in those two specific cases. More precisely, it can be stated that the zero reflectance condition yielded at $162 \mathrm{~nm}, 522 \mathrm{~nm}, 671 \mathrm{~nm}$, and $571 \mathrm{~nm}$ (odd, even layer) respectively. The reason behind is that the wavelength $488 \mathrm{~nm}$ and $514.5 \mathrm{~nm}$ belong to Argon monochromatic signals. Similarly, figure 3(b) shows the reflectance characteristics to realize the generation of Er: YAG monochromatic beams, where two wavelengths such as $2900 \mathrm{~nm}$ and $2940 \mathrm{~nm}$ have been generated.

To sum up ( Figure 3(a) and 3(b)), the wavelengths, 488.0 and $514.5 \mathrm{~nm}$ belong to the visible spectrum whereas the wavelength $2900 \mathrm{~nm}$ and $2940 \mathrm{~nm}$ associated with the short infrared regime. Using the above-said principle, the reflection characteristics have been studied for other wavelengths too. Moreover, the outcomes of other results are lucidly placed in the tabular form in Table 1 ;

Table 1: Information of the proposed photonic structure for a generation the monochromatic beams

\begin{tabular}{|c|c|c|c|c|}
\hline $\begin{array}{l}\text { SL. } \\
\text { No }\end{array}$ & $\begin{array}{c}\text { Name of the } \\
\text { Monochromatic beam }\end{array}$ & $\begin{array}{c}\text { Generating } \\
\text { Wavelength }(\mathrm{nm})\end{array}$ & $\begin{array}{l}\text { Thickness of } \\
\mathrm{SiO}(\mathrm{nm})\end{array}$ & $\begin{array}{l}\text { Thickness of } \\
\text { Si (nm) }\end{array}$ \\
\hline 1 & $\mathrm{He}-\mathrm{Cd}$ & 441.6 & 143 & 49 \\
\hline 2 & $\operatorname{Argon}\left(\mathrm{Ar}^{+}\right)$ & 488.0 & 162 & 52 \\
\hline 3 & & 514.5 & 167 & 57 \\
\hline 4 & \multirow{6}{*}{$\mathrm{He}-\mathrm{Ne}$} & 543.5 & 172 & 63 \\
\hline 5 & & 594.1 & 158 & 73 \\
\hline 6 & & 611.9 & 168 & 71 \\
\hline 7 & & 632.8 & 173 & 75 \\
\hline 8 & & 1152 & 145 & 75 \\
\hline 9 & & 1523 & 193 & 98 \\
\hline 10 & Krypton & 647.1 & 178 & 75 \\
\hline 11 & Ruby & 694.3 & 193 & 78.6 \\
\hline 12 & \multirow{3}{*}{ Nd:YAG } & 946 & 114 & 66 \\
\hline 13 & & 1064 & 135 & 68 \\
\hline 14 & & 1319 & 140 & 65 \\
\hline 15 & Tm:YAG & 2010 & 690 & 479 \\
\hline 16 & Ho:YAG & 2080 & 650 & 508 \\
\hline 17 & \multirow[t]{2}{*}{ Er:YAG } & 2900 & 783 & 675 \\
\hline 18 & & 2940 & 783 & 659 \\
\hline 19 & Er:YSGG & 2790 & 790 & 680 \\
\hline 20 & Iodine & 1315 & 179 & 74 \\
\hline
\end{tabular}

Table 1 provides the complete information to generate the different types of monochromatic beams with the help of silicon based 1D photonic structure. From the above table, it is realized that the thickness of the odd and even layer along with the nature of material plays a vital role in the aforementioned application. After the discussion of 
reflectance, we move to analyze the absorption with the help of the Equation (7-9).Further, the equation (9) depends on the absorption coefficient which is a function of the imaginary refractive index. The imaginary refractive index of the silicon monoxide, silicon, and air material are found at the input signal (violet spectrum). The same can be obtained from the literature [18].Further putting these values of imaginary refractive indices in the Equation (7), (8) and (9), the absorbance, pertaining to the said structure can be found. The computed result affirms that the absorbance of the proposed structure with respect to the said structure is 'zero'. After getting zero reflectance and zero absorbance, we simply move to compute the transmittance of the signal using the following equation

$$
\mathrm{T}=(1-\mathrm{R})(1-\mathrm{A})
$$

Since the values of $\mathrm{R}$ and $\mathrm{A}$ are found to be 'zero' from the aforesaid explanation, the resultant values of the transmittance is 1 , which is confirmed from the Figure 4(a) and 4(b) for the beam of Argon Monochromatic and Er:YAG monochromatic respectively. Like reflectance, simulation for transmittance has also been carried out for entire monochromatic beams but the result for transmittanceof argon and Er:YAG beams are shown in figure 4.
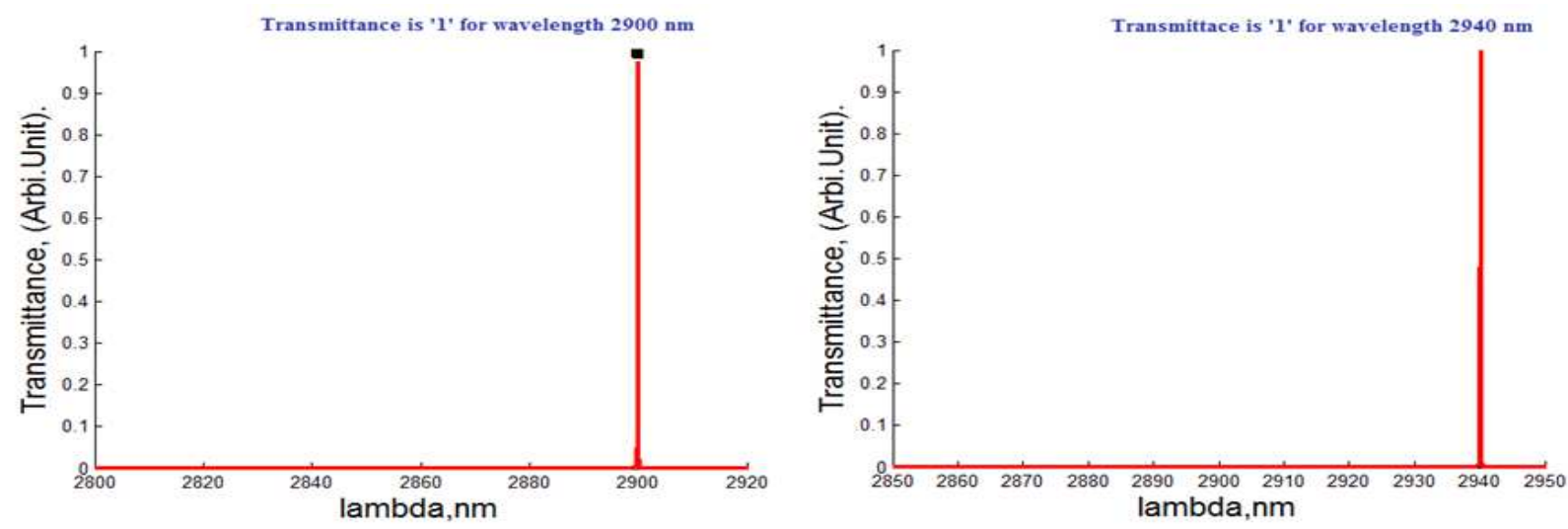

Fig. 4(a) Transmission characteristics of 1D photonic structure for generating argon ( monochromatic) laser beam.
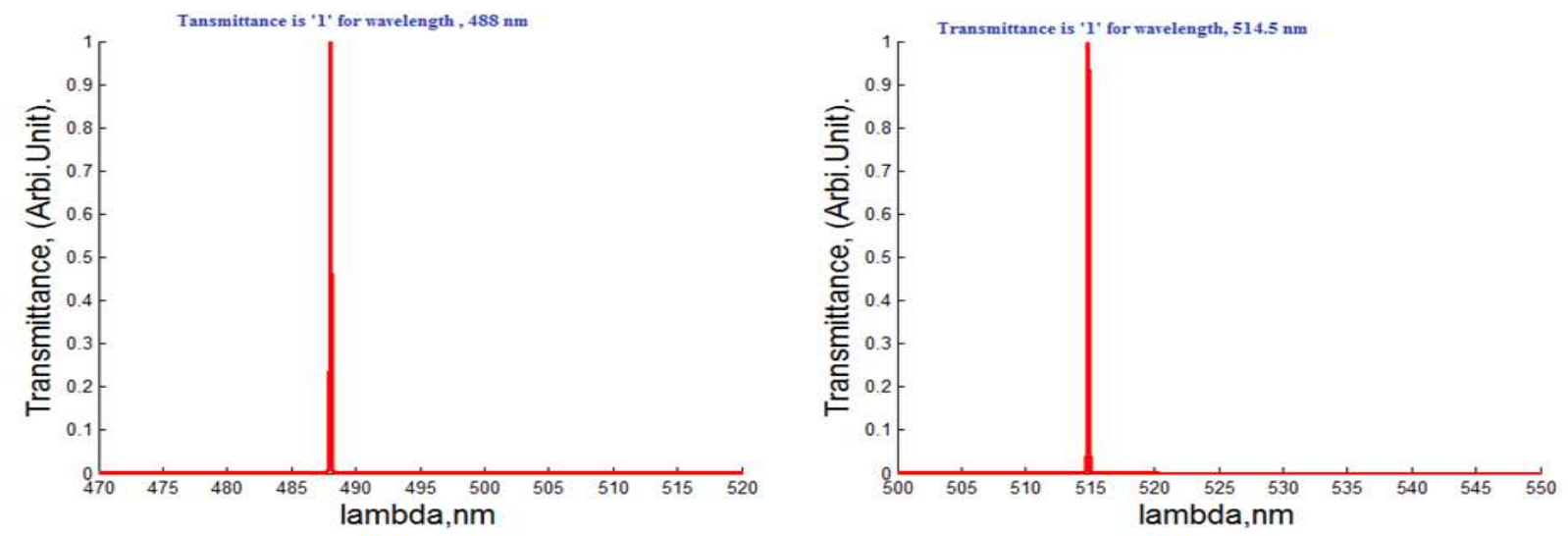

Fig. 4(b) Transmission characteristics of 1D photonic structure for generating Er:YAG laser beam( monochromatic).

It is evident from Figure 4 and Figure 3 that both are quite analogous to each other pertaining to the input signal. After analyzing the above said graphs, transmittance is found to be ' 1 ' $(100 \%)$ at the signal of $488.0 \mathrm{~nm}$ and 514.5 
$\mathrm{nm}$ (visible signal) and $2900 \mathrm{~nm}$ and $2940 \mathrm{~nm}$ (infrared signal) in figure 4(a) and 4(b) respectively, where the transmittance is completely zero at the other wavelengths. Nevertheless, the internal mechanism of the same relies on the effective refractive indices of the silicon monoxide and silicon materials at the signal of incident source. Using the similar explanation and referring the Table 1; it is confirmed that the transmittance corresponding to the signal of $441.6 \mathrm{~nm}, 543.5 \mathrm{~nm}, 594.1 \mathrm{~nm}, 611.9 \mathrm{~nm}, 632.8 \mathrm{~nm}, 1152 \mathrm{~nm}, 1523 \mathrm{~nm}, 647.1 \mathrm{~nm}, 694.3 \mathrm{~nm}, 946 \mathrm{~nm}, 1064 \mathrm{~nm}$, $1319 \mathrm{~nm}, 2010 \mathrm{~nm}, 2080 \mathrm{~nm}, 2900 \mathrm{~nm}, 2940 \mathrm{~nm}, 2790 \mathrm{~nm}, 1315 \mathrm{~nm}$ is found to be 1 or $100 \%$.

\section{CONCLUSION}

Generations of different monochromatic beams in the regime of visible and short IR spectrum are accomplished uniquely in this paper with the help of 1-D silicon-based photonic structure via filtering mechanism. The reflectance characteristics have been meticulously studied through the plane wave expansion method whereas the analysis of absorption is made through numerical treatment. As a matter of fact, the transmittance of the signal bestows the generation of monochromatic beams which could be possible using one dimensional photonic structure. The most fascinating feature of the proposed structure is that the same monochromatic can be applied for different purposes including the medical and communication filed.

\section{REFERENCES}

[1] Afsarinezhad, M., Karimi A. (2019). Plasmonic Nanowire Mono-chromatic using One Dimensional Photonic Crystal Structure. Optik, 181, 246-251

[2] Paul, B.K., Ahmed, K. (2019). Si7N3 material filled novel heptagonal photonic crystal fiber for monochromatic applications. Ceramics International, 45, 1215-1218

[3] Shiri, R., Khalkhali, T.F., Shahrokhabadi, H., Bananej, A. (2019). De-signing one-dimensional magnetized plasma photonic crystals for compensating second- and third-order dispersion effects in ultra-short pulse monochromatics. Optik - International Journal for Light and Electron Optics, 188, 137-146

[4] Tang, M., Park, J., Wang, Z., Chen, S., Jurczak, P., Seeds, A., Liu, H. (2019). Integration of III-V monochromatic on Si for Si photonics. Progress in Quantum Electronics, 66, 1-18

[5] Zakrzewski, A., Patela, S.(2019). Investigation of the monochromatic acetylene sensor based on two-dimensional photonic crystal. Sensors and Actuators A: Physical, 256, 51-58

[6] Amiri, I.S., Sahoo, S.K., Palai, G., Tripathy, S.K. (2019). Generation of '16' type of biomedical monochromatic using a single photonic structure: A new paradigm to operation in medical science. Optik - International Journal for Light and Electron Optics, 197, 163227

[7] Swain, K.P., Palai, G., Moharana, J.K. (2017). Analysis for '101' channels of MUX/DEMUX using grating SOI structure at sub nanometer scale. Optik, 129, 78-82

[8] Swain, K.P., Palai, G. (2018). Photonic structure for embedded application: Realization of optical filter based locking syste. Optik - International Journal for Light and Electron Optics, 169, 344-349

[9] Swain, K.P., Palai. G., Moharana, J.K. (2019). Optical filter based electrical control vis-à-vis cloud data: A new hybrid optoelectronics device for embedded application. Optik - International Journal for Light and Electron Optics, 178, 964 969

[10] Swain, K.P., Palai, G., Moharana, J.K. (2019). Design and implementation of opto-electro decoder using photonic structure: A new application of Li-fi vis-a-vis optical embedded system. Optik - International Journal for Light and Electron Optics, 178, 658-663

[11] Swain, K.P., Nayyar, A., Palai, G. (2019). Prediction of optical specifications through ANN model to design a monochromatic optical filter for all three optical windows. Optik, 198, Article 163314 
[12] Swain, K.P., Subudhi, M., Das, S.R., Palai, G. (2019). Optical Locking System Using Photonic Crystal Structure. ICICCT. Springer, 738-743

[13] Swain, K.P., Das, P.S., Nayak, S.K., Palai, G. (2019). Vehicular control system through VLC: An application of monochromatic optical filters. International Journal of Recent Technology and Engineering (IJRTE), 8, 4508-4512

[14] List of monochromatic types. Date-1/2/2020, From https://en.wikipedia.org/wiki/List_of_monochromatic_types>

[15] AZO optics. Date-10/2/2020, From<https://www.azooptics.com>

[16] Sukhoivanov, I.A., Guryev, I.V. (2009). Physics and Practical Modeling: Photonic Crystals. Springer

[17] Vijaya, M., Rangarajan, G. (2003). Materials Science, Tata McGraw-Hill Education

[18] Schubert, E.F. Materials-Refractive-index-and-extinction-coefficient. From<https://www.ecse.rpi.edu/ schubert/Educational-resources/Materials-Refractive-index-and-extinctioncoefficient.pdf> 